EVS24

Stavanger, Norway, May 13-16, 2009

\title{
A Novel Traction Control without Chassis Velocity for Electric Vehicles
}

\author{
Dejun Yin ${ }^{1}$, Yoichi Hori ${ }^{2}$ \\ ${ }^{1}$ Department of Electrical Engineering, University of Tokyo, Room Ce-503, 4-6-1 Komaba, Meguro, Tokyo, Japan, \\ yin@horilab.iis.u-tokyo.ac.jp \\ ${ }^{2}$ Institute of Industrial Science, University of Tokyo, Room Ce-501, 4-6-1 Komaba, Meguro, Tokyo, Japan, \\ hori@iis-u-tokyo.ac.jp
}

\begin{abstract}
Research on motion control of electric vehicles has progressed considerably, but traction control has not been so sophisticated and practical because the velocity of vehicles and the friction force are immeasurable. This work takes advantage of the features of driving motors to estimate the maximum transmissible torque output in real time based on a purely kinematic relationship, and then proposes an innovative controller to follow the estimated value directly and constrain the torque reference for slip prevention. By comparison with prior control methods, the resulting control design approach is shown to be more effective and robust on an experimental electric vehicle. In addition, a new architecture for a two-dimensional vehicle stability control system is introduced based on the proposed traction control.
\end{abstract}

Keywords: Electric Vehicle, Motion Control, Traction Control, Anti-Slip, Sensorless

\section{Introduction}

Traction control, as a primary control for vehicles, is developed to ensure the effectiveness of the torque output. The key to traction control is anti-slip control, especially for light vehicles because they are more inclined to skid on slippery roads. Traction control must not only guarantee the effectiveness of the torque output to maintain vehicle stability, but also provide some information about tire-road conditions to other vehicle control systems. Moreover, based on the core traction control, more complicated two-dimensional motion control for vehicles can be synthesized by introduction of some information on steering angle, yaw rate, etc. From the viewpoint of the relation between safety and cost, a more advanced traction control synthesis also means lower energy consumption.

However, actual vehicles present a challenge to research on traction control. For example, the real chassis velocity is not available, and the friction force which drives the vehicle is immeasurable. Although anti-lock braking system (ABS) and traction control system (TCS) have been widely applied in conventional internal combustion engine (ICE) vehicles, they need extra control units and hydraulic modules. Furthermore, their performance and universal applicability are hindered by the fact that their control design is based on empirical data rather than general analytical theory [1].

The advantages of electric vehicle offer some new approaches to this problem, as well as to the global problems of the environment and non-renewable resources [2]-[5]. From the viewpoint of motion control, compared with ICE vehicles, the advantages of electric vehicles can be summarized as follows [6]:

1) Quick torque generation

2) Easy torque measurement 
3) Independently equipped motors for each wheel

The torque output of the motor can be easily calculated from the motor current. This merit makes it easy to estimate the driving or braking force between the tire and road surface in real time, which contributes a great deal to application of new control strategies based on road condition estimation. The independently equipped motors provide higher power/weight density, higher redundancy for safety and better dynamic performance [7]-[8].

Some controllers, for example the anti-slip control system based on Model Following Control (MFC), do not need information on chassis velocity or even acceleration sensors. In these systems, the controllers only make use of torque and wheel rotation as input variables for calculation. Fewer sensors contribute not only to lower cost, but also higher reliability and greater independence from driving conditions, which are the most outstanding merits of this class of control systems. Accordingly, research on more practical and more sophisticated anti-slip control based on MFC continues until now. Sakai et al. proposed a primary MFC system for anti-slip control [9]. In their inspiring papers, Saito et al. modified it and proposed a novel stability analysis to decide the maximum feedback gain, and furthermore, took the anti-slip control as a core subsystem and extended it to twodimensional motion control [10]-[11]. Akiba et al. improved the control performance by introduction of back electromotive force, and added a conditional limiter to avoid some of its inherent drawbacks [12]. Nevertheless, these control designs based on compensation have to consider the worst stability case to decide the compensation gain, which impairs the performance of anti-slip control. Furthermore, gain tuning for some specific tire-road conditions

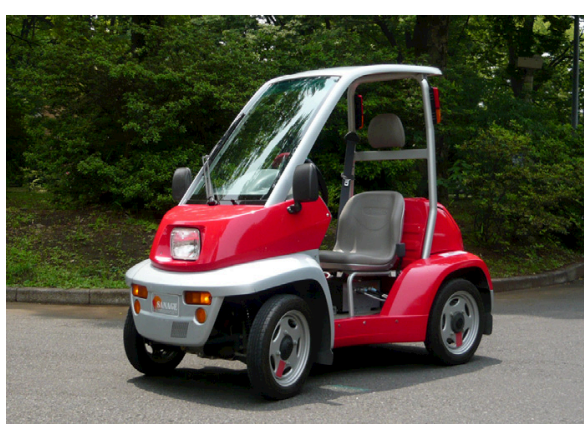

Figure 1: A new experimental electric vehicle also limits the practicability of this method.

Therefore, this paper, making use of the advantages of electric vehicles, focuses on development of a core traction control system based on Maximum Transmissible Torque Estimation (MTTE) that requires neither chassis velocity nor information about tire-road conditions. In this system, use is made of only the torque reference and the wheel rotation to estimate the maximum transmissible torque to the road surface, then the estimated torque is applied for anti-slip control implementation.

The rest of the paper is structured as follows. Section 2 describes an electric vehicle modified for experiments. Section 3 presents a longitudinal model of vehicles, and analyzes the features of anti-slip control. MTTE and a control algorithm based on it are then proposed. Comparing with a prior anti-slip control, Section 4 demonstrates comparative experiments. A discussion on these experimental results follows in Section 5, analyzing the features of the control method.

\section{Experimental Electric Vehicle}

In order to implement and verify the proposed control system, a commercial electric vehicle, COMS, which is made by TOYOTA AUTO BODY Co. Ltd., shown in Fig. 1 was modified to fulfil the experiments' requirements. Each rear wheel is equipped with an Interior Permanent Magnet Synchronous Motor (IPMSM) and can be controlled independently.

As illustrated in Fig. 2, a control computer is added to take the place of the previous ECU to operate the motion control. The computer receives the acceleration reference signal from the acceleration pedal sensor, the forward/backward

Table 1: Specification of COMS3

\begin{tabular}{ll}
\hline Total Weight & $360 \mathrm{~kg}$ \\
Max. Power & $2000 \mathrm{~W} \times 2$ \\
Max. Torque & $100 \mathrm{Nm} \times 2$ \\
Wheel Inertia & $0.5 \mathrm{kgm}^{2} \times 2$ \\
Wheel Radius & $0.22 \mathrm{~m}$ \\
Sampling Time & $0.01 \mathrm{~s}$ \\
Controller & PentiumM 1.8G, 1GB RAM \\
A/D and D/A & 12 bit \\
Shaft Encoder & $36 \mathrm{pulse} /$ round \\
\hline
\end{tabular}




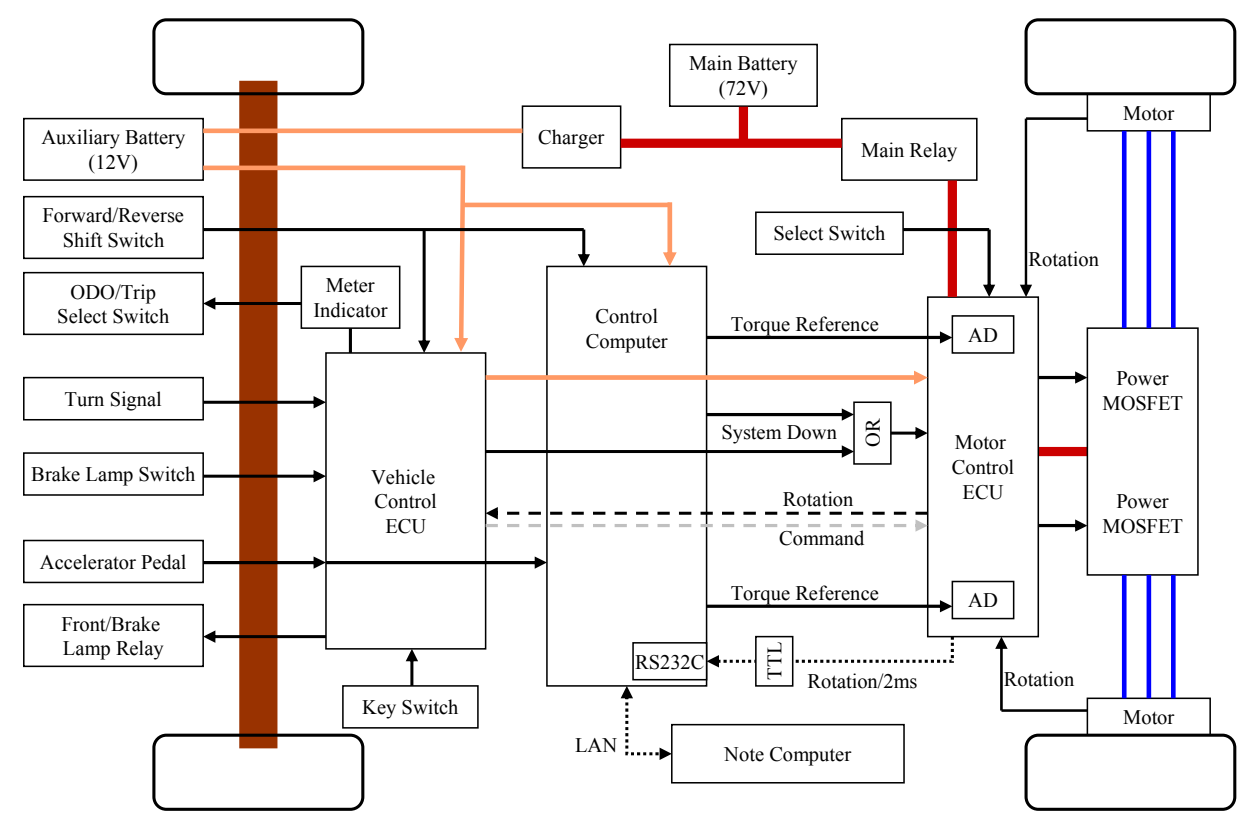

Figure 2: Electrical system of COMS3

signal from the shift switch and the wheel rotation from the inverter. Then, the calculated torque reference of the left and the right rear wheel are independently sent to the inverter by two analog signal lines. Table 1 lists the main specifications.

The most outstanding feature of the modified inverter is that the minimum refresh time of the torque reference is decreased from $10 \mathrm{~ms}$ to $2 \mathrm{~ms}$, which makes it possible to actualize the torque reference more quickly and accurately. The increased maximum rate of change of the torque reference permits faster torque variation for high performance motion control.

\section{Controller Design}

\subsection{MTTE}

Because only longitudinal motion is discussed in this paper, the dynamic longitudinal model of the vehicle can be described by Fig. 3, and the parameter definitions are listed in Table 2.

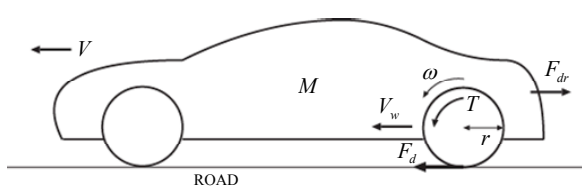

Figure 3: Dynamic longitudinal model of vehicle
Generally, the dynamic differential equations for the calculation of longitudinal motion of the vehicle are described as follows:

$$
\begin{gathered}
J_{w} \dot{\omega}=T-r F_{d} \\
M \dot{V}=F_{d}-F_{d r} \\
V_{w}=r \omega \\
F_{d}(\lambda)=\mu N
\end{gathered}
$$

The interrelationships between the slip ratio and friction coefficient can be described by various formulas. Here, as shown in Fig. 4, the widely adopted Magic Formula [13] is applied to build the vehicle model for the following analysis.

According to (1) and (3), the driving force, i.e. the friction force between the tire and the road surface,

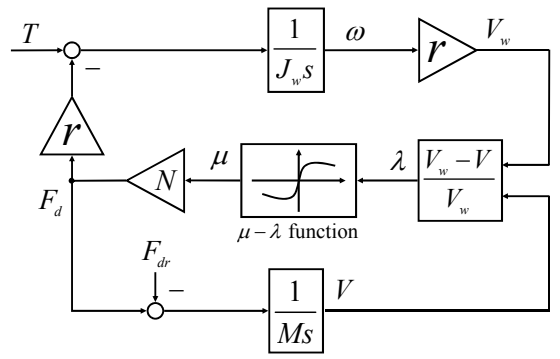

Figure 4: One wheel vehicle model with Magic Formula 
Table 2: Parameter list

\begin{tabular}{cl}
\hline Symbol & Definition \\
\hline$J_{\mathrm{w}}$ & Wheel Inertia \\
$V_{\mathrm{w}}$ & Wheel Circumferential Velocity \\
$\omega$ & Wheel Rotation \\
$T$ & Driving Torque \\
$r$ & Wheel Radius \\
$F_{\mathrm{d}}$ & Friction Force (Driving Force) \\
$M$ & Vehicle Mass \\
$N$ & Vehicle Weight \\
$V$ & Chassis Velocity (Vehicle Velocity) \\
$F_{\mathrm{dr}}$ & Driving Resistance \\
$\lambda$ & Slip Ratio \\
$\mu$ & Friction Coefficient
\end{tabular}

can be calculated as (5). Assuming $T$ is constant, it can be found that the higher $\omega$, the lower $F_{\mathrm{d}}$. In normal road conditions, $F_{\mathrm{d}}$ is less than the maximum friction force from the road and increases as $T$ goes up. However, when slip occurs, $F_{\mathrm{d}}$ equals the maximum friction force that the tire-road relation can provide and cannot increase with $T$. Here, there are only two parameters, $r$ and $J_{\mathrm{w}}$, so $F_{\mathrm{d}}$ is easily calculated in most tire-road conditions.

$$
F_{d}=\frac{T-J_{w} \dot{\omega}}{r}
$$

When slip starts to occur, the difference between the velocities of the wheel and the chassis becomes larger and larger, i.e. the acceleration of the wheel is larger than that of the chassis. Furthermore, according to the Magic Formula, the difference between the accelerations will increase with the slip.

Therefore, the condition that the slip does not start or become more severe is that the acceleration of the wheel is close to that of the chassis. Moreover, considering the $\mu-\lambda$ relation described in the Magic Formula, an appropriate difference between chassis velocity and wheel velocity is necessary to provide the friction force. Accordingly, (6) defines $\alpha$ as a relaxation factor to describe the approximation between the accelerations of the chassis and the wheel. In order to satisfy the condition that slip does not occur or become larger, $\alpha$ should be close to 1 .

$$
\alpha=\frac{\dot{V}^{*}}{\dot{V}_{w}^{*}}, \text { i.e. } \alpha=\frac{\left(F_{d}-F_{d r}\right) / M}{\left(T_{\max }-r F_{d}\right) r / J_{w}}
$$

With a fixed $\alpha$, when the vehicle enters a slippery road, $T_{\max }$ must be reduced adaptively following the decrease of $F_{\mathrm{d}}$ to satisfy (6), the no-slip condition.

Since the friction force from the road is available from (5), the maximum transmissible torque, $T_{\max }$ can be calculated as in (7). This formula indicates that a given $F_{\mathrm{d}}$ allows a certain maximum torque output from the wheel so as not to increase the slip. Here, it must be pointed out that driving resistance, $F_{\mathrm{dr}}$ is assumed to be 0 , which will result in an over evaluation of $T_{\max }$ and consequently impair the anti-slip performance.

$$
T_{\max }=\left(\frac{J_{w}}{\alpha M r^{2}}+1\right) r F_{d}
$$

Finally, the proposed controller can use $T_{\max }$ to constrain the torque reference if necessary.

\subsection{Controller design}

The torque controller is designed as in Fig. 5, in which the limiter with a variable saturation value is expected to realize the control of torque output according to the dynamic situation. Under normal conditions, the torque reference is expected to pass through the controller without any effect. On the other hand, when on a slippery road, the controller can constrain the torque output to be close to $T_{\max }$.

First, the estimator uses the driving torque generated by the inverter-motor system and the rotation speed of the wheel to calculate the friction force, and then estimates the maximum transmissible torque according to (7). Finally, the controller utilizes the estimated torque value as a saturation value to limit the torque reference.

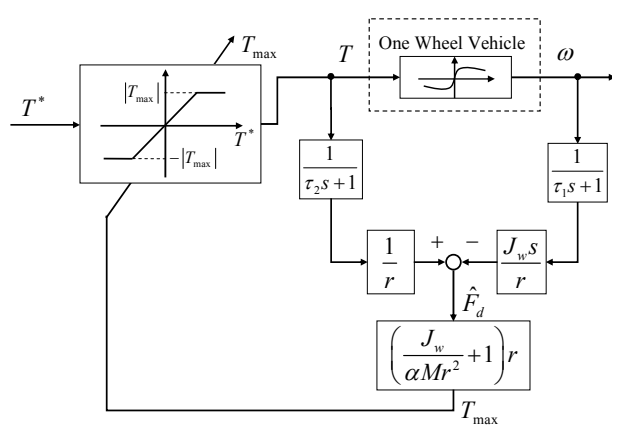

Figure 5: Control system based on MTTE 

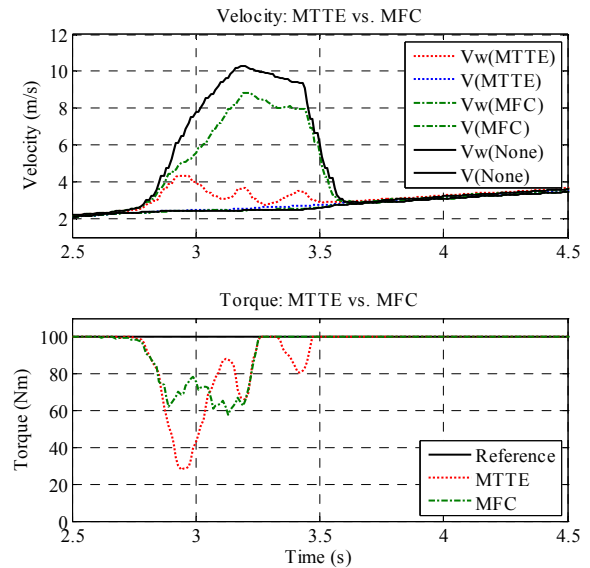

Figure 6: Comparison of experimental results of two control design
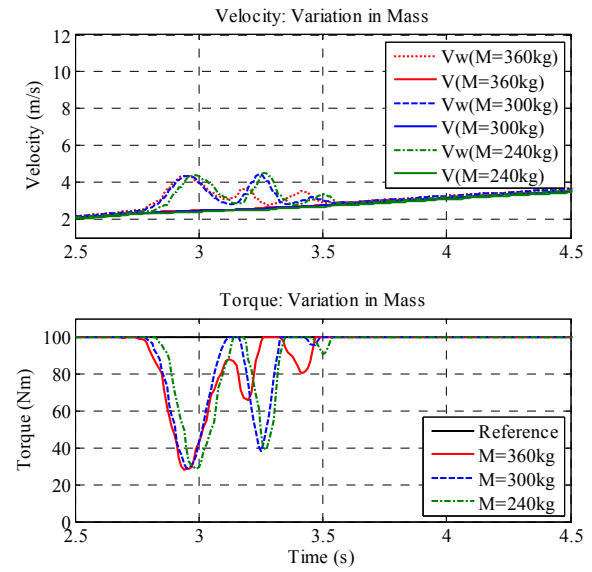

Figure 7: Comparison of experimental results with variation in vehicle mass

Here, although it will cause some phase shift, due to the low resolution of the shaft encoder installed in the wheel, a low pass filter (LPF) with a time constant of $\tau_{1}$ is introduced to smooth the digital signal, $\omega$, for the differentiator which follows. In order to keep the filtered signals in phase, another LPF with a time constant of $\tau_{2}$ is added for $T$. The more precise the shaft encoders are, the smaller the time constants are.

In real experiments, even in normal road conditions, $T_{\max }$ may be smaller than $T^{*}$ due to system delay at the beginning of acceleration, which will cause suddenly commanded
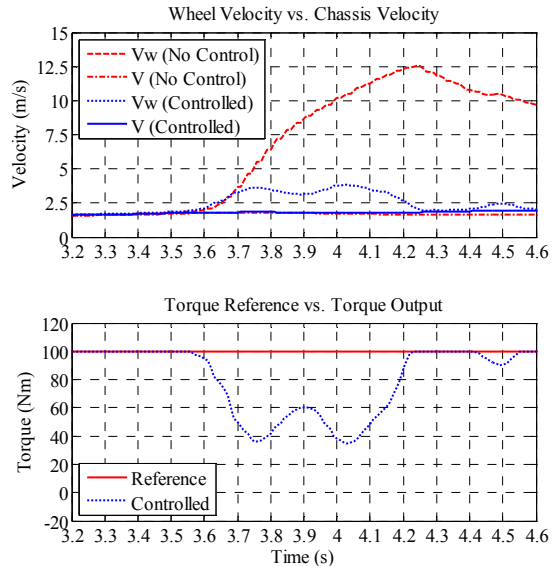

Figure 8: Comparison of experimental results with driving resistance

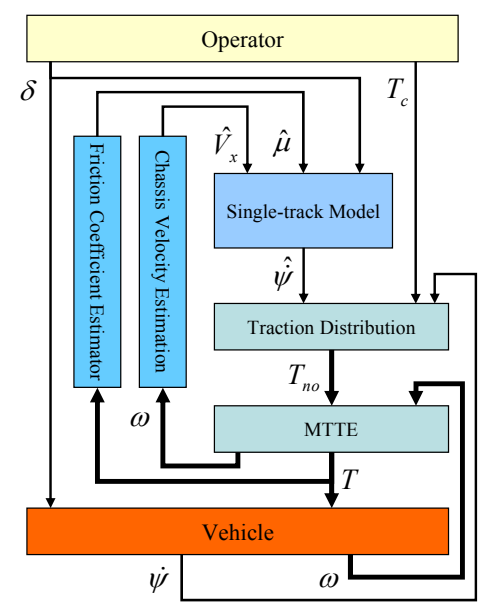

Figure 9: A 2-DOF controller based on MTTE

acceleration to be temporarily constrained by $T_{\max }$ during the acceleration phase. In order to avoid this problem, the increasing rate of $T^{*}$ is amplified as a stimulation to force the under-evaluated $T_{\max }$ to meet the acceleration reference. Here, $T_{\max }$ is used instead of $T_{\max }$ as the input to the controller, whose relation is described by (8). Here, $G$ is a compensation gain. Additionally, the overamplified $T_{\max }$ can be automatically constrained by the following controller. In the following experiments, $G$ was set to 0.1 .

$$
T_{\max }^{\prime}=T_{\max }+\dot{T}^{*} G\left(\dot{T}^{*}>0\right)
$$




\section{Experimental results}

This paper uses the anti-slip control system based on MFC presented in [10] and [11] for the following comparison. In the following experiments, the same parameters of the vehicle are adopted and the gain in the MFC is set to the maximum value that ensures the best control performance while maintaining system stability. In the control based on MTTE, $\alpha$ is fixed as 0.9 , and $\tau_{1}=\tau_{2}=50 \mathrm{~ms}$. The time constant in MFC is set to $50 \mathrm{~ms}$.

Controllers designed as in Fig. 5 were applied to COMS3 for experiments. In these experiments, the slippery road was simulated by an acrylic sheet with a length of $1.2 \mathrm{~m}$ and lubricated with water. The initial velocity of the vehicle was set higher than $1.0 \mathrm{~m} / \mathrm{s}$ to avoid the immeasurable zone of the shaft sensors installed in the wheels. Here, it must be pointed out that in order to detect the chassis velocity, only the left rear wheel is driven by the motor, while the right rear wheel rolls freely to provide a reference value of the chassis velocity for comparison.

Fig. 6 describes the comparison of control performance between the control based on MTTE and MFC, as well as the non-control case.

Because the vehicle mass varies significantly in a real driving environment, In order to evaluate the robustness with variation in vehicle mass, some comparative experiments were performed with different nominal mass in the proposed controller while keeping the real vehicle mass fixed as $360 \mathrm{~kg}$. Fig. 7 provides these comparative results.

Fig. 8 describes the results of the experiment with driving resistance. In these experiments, the driving resistance was simulated as $230 \mathrm{~N}$, corresponding to the air resistance of a BMW 8series running at a speed of $86 \mathrm{~km} / \mathrm{h}$.

\section{Discussion}

Fig. 6 shows that, compared to the no-control case, the difference between the wheel velocity and the chassis velocity does not increase. The estimated maximum transmissible torque is close to the input reference torque for the normal road, and corresponds to the maximum friction force allowed by the slippery tire-road surface.

Fig. 7 and Fig. 8 show that the proposed control system has high robustness to perturbations in vehicle mass and disturbances in driving resistance.
Fig. 6 also shows that, compared with the control based on MFC, MTTE can provide better anti-slip control performance due to its use of a different control philosophy [14]. In fact, in contrast to the control based on MFC, the proposed control method is essentially based on control of wheel behaviour rather than control of the whole chassis behaviour, decreasing the interference between the traction control and the chassis control based on it.

The architecture of a vehicle stability control system based on MTTE is shown in Fig. 9. Here, the steering-wheel angle, the estimated vehicle linear velocity and friction coefficient are used to calculate the nominal yaw rate, then the difference between the measured yaw velocity and the nominal yaw rate acts as the input to the traction distribution module. Finally, the traction distribution module generates the nominal torque reference for the MTTE.

\section{Conclusion}

This paper proposed an estimator of the maximum transmissible torque and applied it to control the driving motors in electric vehicles. The usefulness of the estimator indicated that the motor can act not only as a general actuator, but also as a measurement device because of its inherent features, which provides a good basis for anti-slip control as well as other more advanced motion control systems in vehicles.

The controller designed to co-operate with the estimator can provide higher anti-slip performance in a variety of tire-road conditions while maintaining stability. Comparative experiments with variation of control variables proved the effectiveness and robustness of the proposed control design.

Furthermore, it is possible to construct a twodimensional vehicle stability control system based on the proposed traction control.

\section{References}

[1] Hyeongcheol Lee and Masayoshi Tomizuka, Adaptive Vehicle Traction Force Control for Intelligent Vehicle Highway Systems (IVHSs), IEEE Transaction on Industrial Electronics, Vol. 50, No. 1, pp.37-47, 2003.

[2] K. T. Chau, C. C. Chan, and Chunhua Liu, Overview of Permanent-Magnet Brushless Drives for Electric and Hybrid Electric Vehicles, IEEE Transaction on Industrial Electronics, Vol. 55, No. 6, pp. 2246-2257, 2008. 
[3] Antonio Affanni, Alberto Bellini, Giovanni Franceschini, Paolo Guglielmi, and Carla Tassoni, Battery Choice and Management for New-Generation Electric Vehicles, IEEE Transaction on Industrial Electronics, Vol. 52, No. 5, pp.1343-1349, 2005.

[4] Masao Nagai, The perspectives of research for enhancing active safety based on advanced control technology, Vehicle System Dynamics, Vol. 45, No. 5, pp.413431, 2007.

[5] Naohisa Hashimoto, Manabu Omae, and Hiroshi Shimizu, A Study on Reliable Intelligent Vehicle Driving System by using Features of Electric Vehicles - Lane keeping by Traction-Force-Distribution Control, International Journal of ITS Research, Vol. 1, No. 1, pp.25-31, 2003.

[6] Yoichi Hori, Future Vehicle Driven by Electricity and Control -Research on FourWheel-Motored "UOT Electric March II", IEEE Transaction on Industrial Electronics, Vol. 51, No. 5 pp.954-962, 2004.

[7] Peng He, Yoichi Hori, Optimum Traction Force Distribution for Stability Improvement of $4 W D E V$ in Critical Driving Condition, IEEE International Workshop on Advanced Motion Control, pp.596-601, 2006.

[8] Nobuyoshi Mutoh, Yusuke Takahashi, and Yoshiki Tomita, Failsafe Drive Performance of FRID Electric Vehicles With the Structure Driven by the Front and Rear Wheels Independently, IEEE Transaction on Industrial Electronics, Vol. 55, No. 6, pp. 2306-2315, 2008.

[9] Shin-ichiro Sakai and Yoichi Hori, Advantage of Electric motor for anti skid control of electric vehicle, European Power Electronics Journal, vol. 11, No. 4, pp.26-32, 2001.

[10] Takeo Saito, Hiroshi Fujimoto and Toshihiko Noguchi, Yaw-Moment Stabilization Control of Small Electric Vehicle, Industrial Instrumentation and Control, IEE Japan, pp.83-88, 2002.

[11] Hiroshi Fujimoto, Takeo Saito and Toshihiko Noguchi, Motion Stabilization Control of Electric Vehicle under Snowy Conditions Based on Yaw-Moment Observer, IEEE International Workshop on Advanced Motion Control, pp.35-40, 2004.

[12] Toru Akiba, Ryota Shirato,Takeshi Fujita and Jun Tamura, A study of Novel Traction Control Model for Electric Motor Driven Vehicle, 4th Power Conversion Conference-
NAGOYA, Conference Proceedings, pp.699704, 2007.

[13] Hans B. Pacejka and Egbert Bakker, The Magic Formula Tyre Model, Vehicle System Dynamics, Vol. 21, No. 1, pp.1-18, 1992.

[14] Dejun Yin, Sehoon Oh, and Yoichi Hori, $A$ Novel Traction Control for EV Based on Maximum Transmissible Torque Estimation, IEEE Transaction on Industrial Electronics, Vol. 56, No.6, pp.2086-2094, 2009.

\section{Authors}

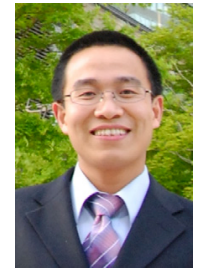

Dejun Yin received the B.S. and M.S degrees in electrical engineering from Harbin Institute of Technology, China in 1999 and 2001 respectively. He received another M.S. degree in electronics engineering from Chiba Institute of Technology, Japan, in 2002, where he worked on embedded control system design in mechatronic engineering for nearly 5 years. In 2009, he received the Ph.D. degree from the Department of Electrical Engineering, the University of Tokyo, Japan.

$\mathrm{He}$ is currently a control specialist at Keio University, Japan, working on the development of vehicle control and motor design for EVs. He is a member of the Institute of Electrical Engineers of Japan, the Society of Automotive Engineers of Japan, and the IEEE Industrial Electronics Society.

Yoichi Hori received Ph.D. degree in electrical engineering from The University of Tokyo in 1983. He became a Professor in 2000. His research fields are control theory and its industrial applications to motion control, mechatronics, robotics, electric vehicles, etc. He is a Fellow of the IEEE, Society of Instrument and Control Engineers, Robotics Society of Japan, Japan Society of Mechanical Engineers, and Society of Automotive

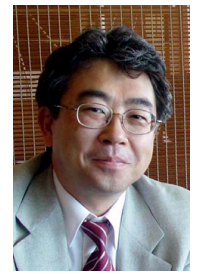
Engineers of Japan, etc. $\mathrm{He}$ is currently the President of the Industry Applications Society of the Institute of Electrical Engineers of Japan, the President of Capacitors Forum, and the Chairman of Motor Technology Symposium of Japan Management Association. 